\title{
NGC 1266 AS A LOCAL CANDIDATE FOR RAPID CESSATION OF STAR FORMATION
}

\author{
Katherine Alatalo ${ }^{1,2}$, Kristina Nyland ${ }^{3}$, Genevieve Graves ${ }^{1,4}$, Susana Deustua ${ }^{5}$, Kristen Shapiro Griffin ${ }^{6}$, \\ Pierre-Alain Duc ${ }^{7}$, Michele Cappellari ${ }^{8}$, Richard M. McDermid $^{9,10,11}$, Timothy A. Davis ${ }^{12}$, Alison F. Crocker ${ }^{13}$, \\ Lisa M. Young ${ }^{3,14}$, Philip Chang ${ }^{15}$, Nicholas Scott ${ }^{16,17}$, Sabrina L. Cales ${ }^{18}$, Estelle Bayet ${ }^{8}$, Leo Blitz ${ }^{1}$, \\ Maxime Bois $^{19}$, Frédéric Bournaud ${ }^{7}$, Martin Bureau ${ }^{8}$, Roger L. Davies ${ }^{8}$, P. T. De ZeeuW ${ }^{10,20}$, \\ Eric Emsellem ${ }^{10,21}$, Sadegh Khochfar ${ }^{22}$, Davor Krajnović ${ }^{23}$, Harald Kuntschner ${ }^{8}$, RafFaella Morganti ${ }^{24,25}$, \\ Thorsten NaAB ${ }^{26}$, Tom Oosterloo ${ }^{24,25}$, Marc Sarzi $^{27}$, Paolo Serra $^{24,28}$, And Anne-Marie WeiJmans ${ }^{29}$ \\ ${ }^{1}$ Department of Astronomy, Hearst Field Annex, University of California, Berkeley, CA 94720, USA \\ ${ }^{2}$ Infrared Processing and Analysis Center, California Institute of Technology, Pasadena, CA 91125, USA \\ ${ }^{3}$ Physics Department, New Mexico Technology, Socorro, NM 87801, USA \\ ${ }_{5}^{4}$ Department of Astrophysical Sciences, Princeton University, Princeton, NJ 08544, USA \\ ${ }^{5}$ Space Telescope Science Institute, 3700 San Martin Drive, Baltimore, MD 21218, USA \\ ${ }^{6}$ Space Sciences Research Group, Northrop Grumman Aerospace Systems, Redondo Beach, CA 90278, USA \\ ${ }^{7}$ Laboratoire AIM Paris-Saclay, CEA/IRFU/SAp-CNRS-Université Paris Diderot, F-91191 Gif-sur-Yvette, Cedex, France \\ ${ }^{8}$ Sub-Department of Astrophysics, Department of Physics, University of Oxford, Denys Wilkinson Building, Keble Road, Oxford OX1 3RH, UK \\ ${ }_{9}^{9}$ Australian Astronomical Observatory, P.O. Box 296, Epping, NSW 1710, Australia \\ ${ }^{10}$ Department of Physics and Astronomy, Macquarie University, NSW 2109, Australia \\ ${ }^{11}$ Gemini Observatory, Northern Operations Center, 670 N. A'ohoku Place, Hilo, HI 96720, USA \\ 12 European Southern Observatory, Karl-Schwarzschild-Street 2, D-85748 Garching, Germany \\ ${ }^{13}$ Department of Physics and Astronomy, University of Toledo, Toledo, OH 43606, USA \\ ${ }^{14}$ National Radio Astronomy Observatory, Socorro, NM 87801, USA \\ ${ }^{15}$ Department of Physics, University of Wisconsin-Milwaukee, Milwaukee, WI 53201, USA \\ ${ }^{16}$ Centre for Astrophysics and Supercomputing, Swinburne University of Technology, P.O. Box 218, Hawthorn VIC 3122, Australia \\ ${ }^{17}$ Sydney Institute for Astronomy (SIfA), School of Physics, The University of Sydney, NSW 2006, Australia \\ ${ }^{18}$ Department of Astronomy, Faculty of Physical and Mathematical Sciences, Universidad de Concepción, Casilla 160-C, Concepción, Chile \\ ${ }^{19}$ Observatoire de Paris, LERMA and CNRS, 61 Avenue de l'Observatoire, F-75014 Paris, France \\ ${ }^{20}$ Sterrewacht Leiden, Leiden University, Postbus 9513, 2300-RA Leiden, The Netherlands \\ ${ }^{21}$ Université Lyon 1, Observatoire de Lyon, Centre de Recherche Astrophysique de Lyon and Ecole Normale Supérieure de Lyon, 9 Avenue \\ Charles André, F-69230 Saint-Genis Laval, France \\ ${ }^{22}$ Max-Planck Institut für Extraterrestrische Physik, P.O. Box 1312, D-85478 Garching, Germany \\ ${ }^{23}$ Leibniz-Institut für Astrophysik Potsdam (AIP), An der Sternwarte 16, D-14482 Potsdam, Germany \\ ${ }^{24}$ Netherlands Institute for Radio Astronomy (ASTRON), Postbus 2, 7990-AA Dwingeloo, The Netherlands \\ ${ }^{25}$ Kapteyn Astronomical Institute, University of Groningen, Postbus 800, 9700-AV Groningen, The Netherlands \\ ${ }^{26}$ Max-Planck-Institut für Astrophysik, Karl-Schwarzschild-Street 1, D-85741 Garching, Germany \\ ${ }^{27}$ Centre for Astrophysics Research, University of Hertfordshire, Hatfield, Herts AL1 9AB, UK \\ ${ }^{28}$ CSIRO Astronomy and Space Science, Australia Telescope National Facility, P.O. Box 76, Epping, NSW 1710, Australia \\ ${ }^{29}$ Dunlap Institute for Astronomy and Astrophysics, University of Toronto, 50 St. George Street, Toronto ON M5S 3H4, Canada \\ Received 2013 May 30; accepted 2013 November 25; published 2013 December 23
}

\begin{abstract}
We present new Spectrographic Areal Unit for Research on Optical Nebulae (SAURON) integral-field spectroscopy and Swift Ultraviolet Optical Telescope (UVOT) observations of molecular outflow host galaxy NGC 1266 that indicate NGC 1266 has experienced a rapid cessation of star formation. Both the SAURON maps of stellar population age and the Swift UVOT observations demonstrate the presence of young ( $<1 \mathrm{Gyr})$ stellar populations within the central $1 \mathrm{kpc}$, while existing Combined Array for Research in Millimeter-Wave Astronomy CO(1-0) maps indicate that the sites of current star formation are constrained to only the inner few hundred parsecs of the galaxy. The optical spectrum of NGC 1266 from Moustakas \& Kennicutt reveal a characteristic poststarburst (K+A) stellar population, and Davis et al. confirm that ionized gas emission in the system originate from a shock. Galaxies with $\mathrm{K}+\mathrm{A}$ spectra and shock-like ionized gas line ratios may comprise an important, overlooked segment of the poststarburst population, containing exactly those objects in which the active galactic nucleus (AGN) is actively expelling the star-forming material. While AGN activity is not the likely driver of the poststarburst event that occurred $500 \mathrm{Myr}$ ago, the faint spiral structure seen in the Hubble Space Telescope Wide-field Camera $3 Y$-, Jand $H$-band imaging seems to point to the possibility of gravitational torques being the culprit. If the molecular gas were driven into the center at the same time as the larger scale galaxy disk underwent quenching, the AGN might be able to sustain the presence of molecular gas for $\gtrsim 1 \mathrm{Gyr}$ by cyclically injecting turbulent energy into the dense molecular gas via a radio jet, inhibiting star formation.
\end{abstract}

Key words: galaxies: active - galaxies: evolution - galaxies: individual (NGC 1266)

Online-only material: color figures

\section{INTRODUCTION}

The present-day galaxy population has a bimodal color distribution with a genuine lack of intermediate-color galaxies (Strateva et al. 2001; Baldry et al. 2004). The lack of galaxies in the "green valley" suggests that galaxies migrate rapidly between the blue cloud and red sequence, requiring a rapid quenching of star formation (SF; Bell et al. 2004; Faber et al. 2007). Recent simulations have suggested that active galactic nuclei (AGNs) may be capable of shutting down SF by heating 
and driving out gas (Springel et al. 2005; Hopkins et al. 2005; Croton et al. 2006; Debuhr et al. 2012). While circumstantial evidence for the quenching of SF via AGN feedback exists (Schawinski et al. 2007), direct evidence has been scarce. There are promising candidates for AGN-driven SF quenching in quasar hosts at $z \sim 2$ (Nesvadba et al. 2008; Cano-Díaz et al. 2012), but only limited information can be obtained from such distant objects. In the more local universe, the low-redshift quasar host Markarian 231 has recently been shown to exhibit a massive molecular outflow (Feruglio et al. 2010; Fischer et al. 2010; Aalto et al. 2012a). However, due to the high current $\mathrm{SF}$ rate, it is difficult to distinguish between a starburst and an AGN-driven origin for the outflow in this system. Other nearby galaxies with candidate AGN-driven molecular outflows such as M51 (Matsushita et al. 2007), NGC 3801 (Das et al. 2005; Hota et al. 2012), and NGC 1377 (Aalto et al. 2012b) have been similarly controversial, with compact starbursts or the effects of a recent major merger potentially dominating over any AGN feedback. Thus, examples of gas-rich systems unaltered by recent major mergers or strong starbursts are needed to better understand the role of AGN feedback in the SF history of local galaxies.

Poststarburst galaxies (i.e., $\mathrm{K}+\mathrm{A}$ and $\mathrm{E}+\mathrm{A}$ galaxies; Dressler \& Gunn 1983; Zabludoff et al. 1996; Quintero et al. 2004; Goto 2005) may be an ideal demographic to study in the search for evidence of direct AGN-driven SF quenching. These galaxies tend to have undergone a rapid ( $<1 \mathrm{Gyr})$ cessation of SF (although a rapid onset of SF is also capable of forming poststarbursts; Falkenberg et al. 2009) and commonly lie in the green valley of the galaxy color-magnitude distribution. While some poststarburst galaxies show obvious signs of disruption and are likely the products of recent major mergers, the reason behind the abrupt halt of SF in others is less clear (Cales et al. 2011). Proposed methods of SF quenching in nonmerging systems include strangulation and ram pressure stripping (as a gas-rich galaxy falls into a cluster environment and experiences tidal effects and winds, respectively; Evrard 1991; Fujita 1998; Bekki et al. 2002; Boselli \& Gavazzi 2006), harassment (in which the gravitational potential of a galaxy is perturbed by its cluster neighbors; Icke 1985; Mihos 1995; Bekki 1998; Moore et al. 1996), tidal stripping (tidal disruption of cold gas due to nearby galaxies, like what is seen in Hickson Compact Groups; Verdes-Montenegro et al. 2001), morphological quenching (the tendency of the molecular gas within a bulge-dominated system to be too stable against gravitational collapse to efficiently form stars; Martig et al. 2009), and AGN-driven feedback in the radiative (ionization, heating, and radiation pressure; Ciotti \& Ostriker 2007) or mechanical mode (nuclear winds and jets; Ciotti et al. 2010). Strangulation, ram pressure stripping, and harassment require a dense cluster environment; tidal stripping requires a group environment and therefore cannot explain solitary poststarbursts; and morphological quenching should not generate the massive molecular outflows observed in some nearby galaxies. Thus, isolated poststarburst galaxies with actively outflowing gas provide snapshots of a critical phase of a galaxy's journey from star-forming to the red sequence.

The discovery of the massive molecular outflow $\left(M_{\text {outflow }}=\right.$ $2.4 \times 10^{7} M_{\odot}, \dot{M}=13 M_{\odot} \mathrm{yr}^{-1}$ ) in NGC 1266 was originally reported in Alatalo et al. (2011). Recently, Davis et al. (2012) also presented their work on integral-field spectroscopic observations of the ionized gas in the nucleus of NGC 1266 and showed that the outflow is truly multiphase, consisting of ionized and atomic gas, molecular gas, X-ray emitting plasma, and
Table 1

HST WFC3 Observations (Program 12525, Three Orbits)

\begin{tabular}{lllll}
\hline \hline Obs ID & Instrument & Channel & Filter & $\begin{array}{c}\text { Exp. Time } \\
(\mathrm{s})\end{array}$ \\
\hline ibr702c6q & WFC3 & IR & F160W & 449 \\
ibr702c7q & WFC3 & IR & F140W & 449 \\
ibr702c9q & WFC3 & IR & F110W & 399 \\
ibr702cbq & WFC3 & IR & F160W & 449 \\
ibr702cdq & WFC3 & IR & F140W & 449 \\
ibr702cfq & WFC3 & IR & F110W & 399 \\
\hline
\end{tabular}

radio emitting plasma. Finally, Nyland et al. (2013) report on the discovery of a high brightness temperature Very Long Baseline Array (VLBA) point source within the nucleus of the galaxy, providing definitive evidence of the presence of an AGN in the system. The distance to NGC 1266 is taken from ATLAS ${ }^{3 \mathrm{D}}$, $29.9 \mathrm{Mpc}$, for which $1^{\prime \prime}=145 \mathrm{pc}$.

Here we report on the stellar population of NGC 1266, particularly how it has changed with time, how that change might relate to poststarburst objects in general, and how we might find other objects undergoing similar events. In Section 2, we describe the observations and data reduction of the Hubble Space Telescope (HST) Wide-field Camera 3 (WFC3), the Swift Ultraviolet Optical Telescope (UVOT), Spectrographic Areal Unit for Research on Optical Nebulae (SAURON) and the $2.3 \mathrm{~m}$ Bok Telescope at Kitt Peak. In Section 3, we show that NGC 1266 contains a poststarburst-like stellar population, and we discuss the implications of NGC 1266's reclassification as a poststarburst galaxy. In Section 5, we summarize our results and suggest future directions for NGC 1266 studies.

\section{OBSERVATIONS AND DATA REDUCTION}

\subsection{Hubble Space Telescope (HST)}

Visible and infrared images of NGC 1266 were obtained with the Hubble WFC3 instrument on the HST in 2011 December. Table 1 lists the HST datasets identification, instrument, channel, filter, and exposure time. All images are full frame and were processed with the standard reduction pipeline CALWF3. Cleaned images were coadded, registered, and scaled to a common pixel scale of $0{ }^{\prime} 13$ pixel $^{-1}$ with MULTIDRIZZLE. The resulting drizzled images were flux calibrated and appear in Figure 5.

\subsection{Swift Ultraviolet Optical Telescope (UVOT)}

NGC 1266 was observed with the Swift UVOT (Roming et al. 2005) under Target-of-Opportunity program 31376 on 2009 March 15 and 17 for $9840 \mathrm{~s}$ and $14220 \mathrm{~s}$ in the nearUV ( $\lambda 2600)$ and far-UV( $\lambda 1928)$ bands, respectively. Data were reduced using the automatic pipeline and coadded using the Swift task UVOTIMSUM from the HEASOFT package ${ }^{30}$ (Breeveld et al. 2010). The resultant images were then sky subtracted, and the sky root mean square noise was determined using the IDL DAOPHOT task SKY. All discussions hereafter focus on the far-UV ( $\lambda 1928)$ data.

\subsection{SAURON}

The SAURON is an integral-field spectrograph at the William Herschel Telescope (Bacon et al. 2001). The SAURON data of NGC 1266 were taken on the nights of 2008 January 10 and 11

\footnotetext{
30 HEASOFT software can be found at

http://heasarc.gsfc.nasa.gov/docs/software/lheasoft/.
} 
as part of the ATLAS ${ }^{3 \mathrm{D}}$ observing campaign (Cappellari et al. 2011a). SAURON covers the wavelength range 4810-5350 $\AA$ with a spectral resolution of $105 \mathrm{~km} \mathrm{~s}^{-1}$. The SAURON observations were reduced using the standard ATLAS ${ }^{3 \mathrm{D}}$ pipeline (Cappellari et al. 2011a), and the processed data cubes were Voronoi binned (Cappellari \& Copin 2003).

In order to measure the absorption line strengths, the data were first processed using GANDALF (Sarzi et al. 2006), which provided the best-fit combination of absorption and emission lines. The emission contribution was then subtracted from the SAURON spectrum. All details of the $\mathrm{H} \beta$ absorption $\left(\mathrm{H} \beta_{\mathrm{abs}}\right)$ and single stellar population (SSP) modeling will be presented in R. M. McDermid et al. (2014, in preparation). To apply astrometry to the SAURON images, the peak of the integrated SAURON map was matched to the peak of the $V$-band image from the Spitzer Infrared Nearby Galaxy Survey (Kennicutt et al. 2003). Since the SAURON wavelength range is nearest to the $V$-band, this provided a reasonable match for the astrometry, allowing for world coordinate system info based on the match to the $V$-band data to be written into the SAURON $\mathrm{H} \beta_{\text {abs }}$ and stellar age headers.

\subsection{Long-slit Spectroscopy}

We also provide a new analysis of long-slit spectroscopy originally published in Moustakas \& Kennicutt (2006). These data were originally obtained at the $2.3 \mathrm{~m}$ Bok Telescope on Kitt Peak using the Boller and Chivens spectrograph, providing for spectral coverage between 3600 and $6900 \AA$ with $2.75 \AA$ pixels and a full-width at a half-maximum resolution of $8 \AA$ through a 2 .'5 wide by 3.3 long slit. A spectroscopic drift scan technique (scanning the slit across the galaxy while integrating) was used to construct an integrated spectrum. The drift scan length perpendicular to the slit for NGC 1266 was 55", with a total exposure time of $2400 \mathrm{~s}$. The nuclear spectrum of NGC 1266 was obtained based on 5 minute exposures using a fixed 2 ". $5 \times 2$ ".5 slit aperture. The final flux-calibrated spectra of the nucleus and integrated data for NGC 1266 were delivered in a FITS table to be analyzed for this article, and further details on the reduction and analysis techniques are available in Moustakas \& Kennicutt (2006). The spectral range of these observations allows for a more robust determination of stellar populations by providing a much larger set of Balmer absorption lines than the SAURON observations, which only include $\mathrm{H} \beta$.

\section{METHODS AND ANALYSIS}

\subsection{The Stellar Composition of NGC 1266}

To determine the age composition of stars within NGC 1266, we used the spectra originally published in Moustakas \& Kennicutt (2006). We masked emission lines known to be part of the shock from Davis et al. (2012) at [O II] $\lambda 3727$, [O III] $\lambda 5007$, $\mathrm{H} \alpha, \mathrm{H} \beta,\left[\mathrm{N}_{\mathrm{II}}\right] \lambda 6583$, and [S II] $\lambda 6716$, as well as visible $\mathrm{Na} \mathrm{D}$ absorption and a sky-line $(\lambda 5577)$. We then used the Penalized Pixel-Fitting (pPXF) IDL procedure (Cappellari \& Emsellem 2004) to fit a set of stellar population templates from the MIUSCAT library (Vazdekis et al. 2012), which is an update on the original MILES library (Falcón-Barroso et al. 2011), spanning $-0.71<[Z / H]<+0.20$ linearly in metallicity and logarithmically spanning $63 \mathrm{Myr}$ to $17.8 \mathrm{Gyr}$ logarithmically in age (Vazdekis et al. 2010). Four initial mass functions (IMFs) were used to derive the differences in fractions across IMF choices, including unimodal (Salpeter 1955), bimodal (Vazdekis et al. 2010), and finally universal Kroupa and revised
Kroupa (Kroupa 2001). The spectra fits were also done with pPXF using the MILES library (Falcón-Barroso et al. 2011) in order to check the efficacy of the models and search for large diversions between model fits, with similarly selected $[\mathrm{Z} / \mathrm{H}]$ and age parameters. The MILES models derived equivalent mass fractions in each IMF as found by the MIUSCAT models.

In order to gauge the different possible SF histories that could be present in NGC 1266, pPXF was run with two different assumptions. The first assumption is that NGC 1266 has had a monolithic SF history, leading to a much smoother stellar population weight distribution, which was created using line regularization (setting the REGUL parameter in pPXF; Cappellari \& Emsellem 2004). Setting a nonzero REGUL keyword has the effect of pushing a SF history toward a more linear shape in age and $Z$ space, and effectively providing the smoothest fit among the many degenerate solutions that fit the data equally well. This linearization is also able to allow us to explore the degeneracy among the various stellar population models. The second was assuming a bursty SF history (setting REGUL $=0$ ), which the multiple young subcomponents of NGC 1266 seem to point to. Both sets of assumptions create a bimodal stellar distribution, and both the bursty and smoothly varying SF histories produced the same light fraction for young versus old stars when fit with the MIUSCAT models. Figures 1 and 2 show the flux-calibrated long-slit spectra, the MIUSCAT universal Kroupa model fits, as well as the light fraction weighting given to the component stellar ages and metallicities fitted by pPXF. These figures indicate that the distribution of models appears to be bimodal, with a "young" ( $<2 \mathrm{Gyr})$ and "old" (>10 Gyr) stellar population. As there is degeneracy in the $>10$ Gyr stellar population models, the weighting of stellar models are driven toward the edge of the distribution in NGC 1266, which has long been known to host (at least in part) a large mass of old stars. Although we are unable to pinpoint exactly the metallicity and age of the "older" stellar population, pPXF is able to provide a good estimate of the relative light contributions to the spectra. Table 2 summarizes the results of fitting the integrated and nuclear spectra to the MIUSCAT models with four IMF choices. The mass fractions were derived based on the output weights from the bursty SF $($ REGUL $=0)$ models, and light fractions were derived based on the mass-to-light ratios in $V$-band derived directly from the IMFs used. Finally, a classical A/K fit was also run, limiting pPXF to two templates, solar metallicity A $(0.3 \mathrm{Gyr})$ and $\mathrm{K}$ (10.0 Gyr) templates, which are derived from the MIUSCAT Kroupa universal IMF set of stellar population models.

In order to run a classical $\mathrm{K}+\mathrm{A}$ analysis, first described in Dressler \& Gunn (1983) and later in Quintero et al. (2004), we constrained the models available to pPXF to A-star $(0.3 \mathrm{Gyr})$ and K-star $(10.0 \mathrm{Gyr})$ models with solar metallicity $([\mathrm{Z} / \mathrm{H}]=$ $0.0)$. The inferred contribution of young stars to the spectrum is larger when we limit our models to K+A models (see Table 2). We find that the A-star mass contribution to all spectra is $\approx 10 \%$, with $68 \%-85 \%$ of the light originating from the A-stars. The classical $\mathrm{K}+\mathrm{A}$ fitting seems to have slightly overestimated the total contribution of young stars within the NGC 1266 spectrum. It is possible that constraining fits to these templates in other poststarburst searches might have overestimated the total contribution of young stars as well.

Using the MIUSCAT results from Figures 1 and 2, the average age of the "young" stellar population is approximately $0.8 \mathrm{Gyr}$ in the outer regions and $\approx 0.5 \mathrm{Gyr}$ in the nucleus, which is older than an A-star but still much younger than the "old" stellar 

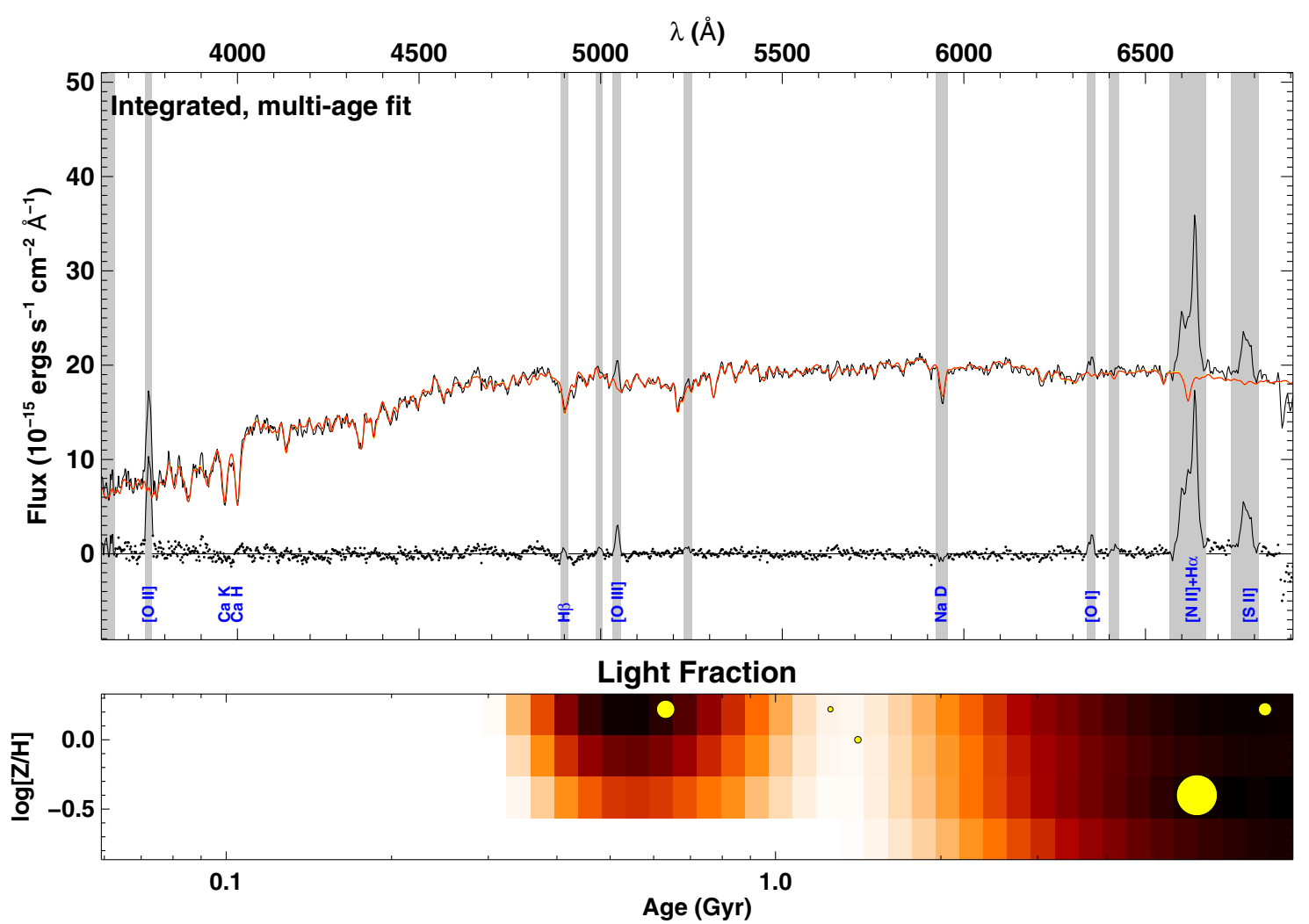

Figure 1. Top: integrated spectra (black) from 3800-6000 A are overlaid with the stellar model fits from MIUSCAT multiplied by a weighting supplied by pPXF for both bursty (yellow) and continuous (red) modes, using a universal Kroupa initial mass function (Kroupa 2001). Residuals (black points) and masked portions of the spectra (shaded gray) are also included, and well-known lines are identified in blue. The parameters of the integrated fit did not vary between different libraries (MILES or MIUSCAT; Vazdekis et al. 2010), although they did depend on the chosen IMF (see Table 2). Bottom: the relative age-metallicity weights derived from a pPXF fit to a continuous SF history using a universal Kroupa IMF (controlled by the line regularization weighting given through the REGUL keyword in pPXF; Cappellari \& Emsellem 2004) for the spectrum. The redscale represents a linear light fraction weighting, with darker regions tending to have higher weights. The results of a bursty SF history light fraction weights are shown as yellow points (of which there are five), with their size representing the relative contribution of each age and metallicity to the fit. Light fraction was derived using the $V$-band mass-to-light ratio for each metallicity and age bin using the universal Kroupa IMF.

(A color version of this figure is available in the online journal.)

population. It is possible that constraining fits to just these two templates is far too simplistic for understanding the SF history (and possibly the poststarburst phase) for galaxies.

Future work utilizing broadband $H S T$ imaging to create a high-resolution multicolor map of NGC 1266, in conjunction with spectral energy distribution modeling of stellar populations (da Cunha et al. 2008), will be able to create a map of the SF history within the galaxy, thus providing a much more detailed look at the way in which SF has shut down throughout the disk of NGC 1266. New, deep, high-resolution data will be capable of tracing star-forming regions in the molecular disk as well as the environment surrounding the central AGN.

\subsection{Spatial Distribution of Star Formation}

Because of the compact nature of the molecular gas, we examine the comparative extent of both current and recent SF to infer the progression of the SF in NGC 1266. The molecular gas not involved in an outflow is concentrated in a central disk of radius $<250 \mathrm{pc}$ (Alatalo et al. 2011), so any current or future SF activity must be concentrated within this radius.

In contrast, young stellar populations, as traced by stellar absorption line strengths (Figure 3; R. M. McDermid et al. 2014, in preparation), are found throughout the SAURON field-of-view $\left(40^{\prime \prime}=6 \mathrm{kpc}\right)$. A map of the SSP-equivalent ages in NGC 1266 indicates that the youngest stellar populations are concentrated toward the center of the galaxy, particularly within the central $5^{\prime \prime}-6$ " (the central kpc; Figure 3). The measured SSP ages range from approximately $1.1 \mathrm{Gyr}$ in the central kpc to about $2 \mathrm{Gyr}$ at larger radii, agreeing well with the pPXF fit to the Moustakas \& Kennicutt (2006) spectrum discussed in Section 3.1. These SSP ages represent a luminosity-weighted combination of young and old stellar populations, so the actual ages of the young stellar populations cannot be directly determined. The UV emission, known to trace young (as opposed to nascent) stars (Kennicutt 1998) also shows that there are young stars over a larger scale than the molecular gas (see Figure 4), out to a radius of $2 \mathrm{kpc}$.

The combined stellar population data and SF tracers paint an intriguing picture of NGC 1266. Less than 1 Gyr ago, SF was occurring on large scales ( $\gtrsim 6 \mathrm{kpc}$, the edge of the SAURON fieldof-view). However, any ongoing or future SF will be fueled by the available molecular gas and thus only occur in the central 250 pc. In NGC 1266, we may be observing an object just as it is transitioning into a poststarburst phase in which widespread SF has ceased over the course of $\lesssim 1$ Gyr. Central SF is expected to be suppressed rapidly ( $<100 \mathrm{Myr}$; Alatalo et al. 2011) in the center of NGC 1266, corresponding with the ignition of the AGN.

\subsection{Star Formation History of NGC 1266}

In order to determine whether NGC 1266 would classically be considered a poststarburst galaxy, we followed the definition of Quintero et al. (2004) that $\mathrm{K}+\mathrm{A}$ galaxies have $\mathrm{A} / \mathrm{K}$ light fraction ratios $\gtrsim 0.2$. The $\mathrm{A} / \mathrm{K}$ fraction for the nucleus of NGC 1266 is 5.7 (it is 2.1 for the integrated spectrum). 

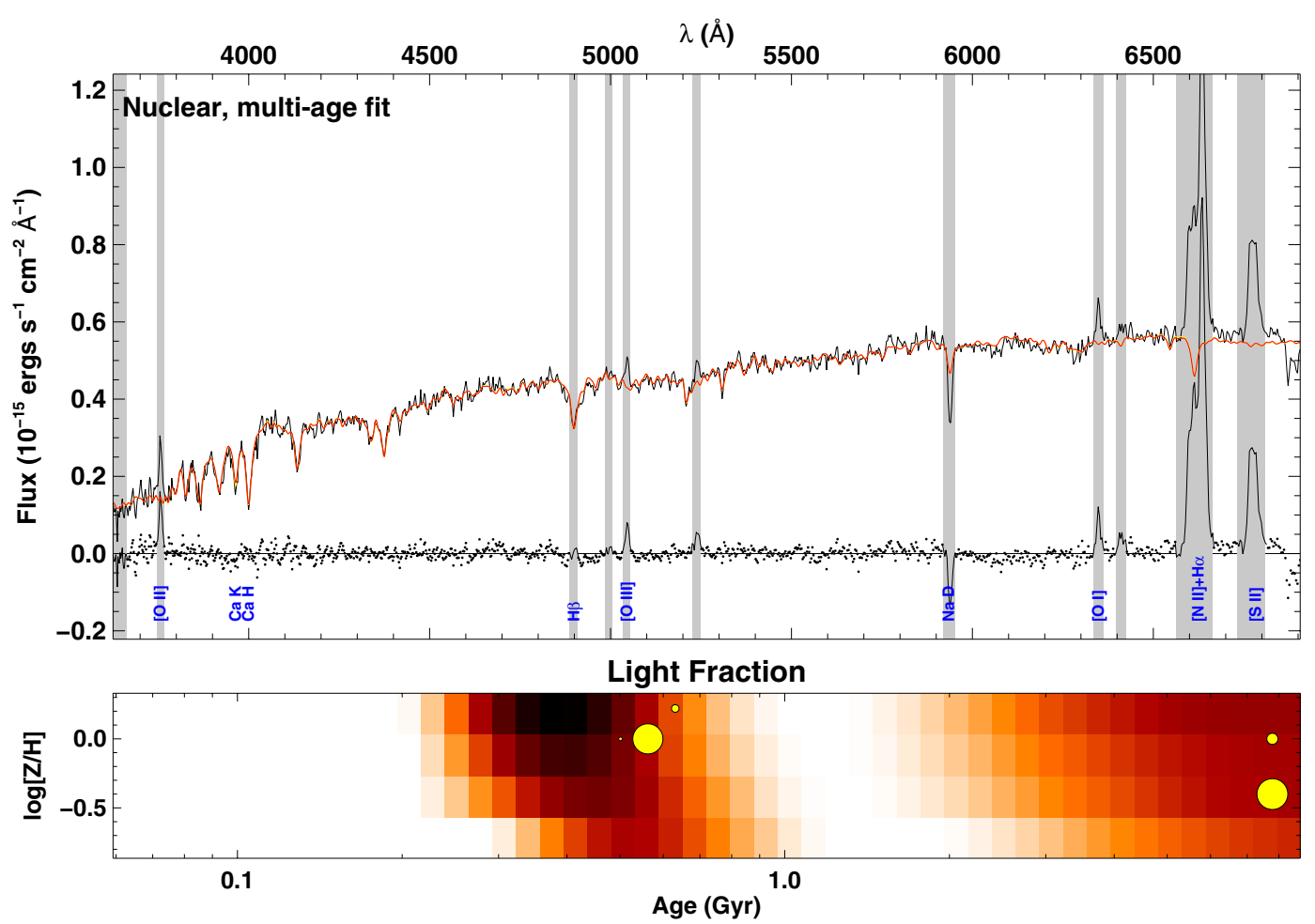

Figure 2. Top: nuclear spectra (black) from 3800-6000 $\AA$ are overlaid with the stellar model fits from MIUSCAT multiplied by a weighting supplied by pPXF for both bursty (yellow) and continuous (red) modes, using a universal Kroupa initial mass function (Kroupa 2001). Residuals (black points) and masked portions of the spectra (shaded gray) are also included, and well-known lines are identified in blue. The parameters of the nuclear fit did not vary between different libraries (MILES or MIUSCAT; Vazdekis et al. 2010), although they did depend on the chosen IMF (see Table 2). Bottom: the relative age-metallicity weights derived from a pPXF fit to a continuous SF history using a universal Kroupa IMF (controlled by the line regularization weighting given through the REGUL keyword in pPXF; Cappellari \& Emsellem 2004) for the spectrum. The redscale represents a linear light fraction weighting, with darker regions tending to have higher weights. The results of a bursty SF history light fraction weights are shown as yellow points (of which there are six), with their size representing the relative contribution of each age and metallicity to the fit. Light fraction was derived using the $V$-band mass-to-light ratio for each metallicity and age bin using the universal Kroupa IMF.

(A color version of this figure is available in the online journal.)

NGC 1266 would thus be classified as a poststarburst, confirmed by the fraction of $<2$ Gyr stars from the multicomponent stellar population fit.

The poststarburst classification requires both a young stellar population and a reduction of current SF. The nucleus of NGC 1266 has an A/K stellar ratio that satisfies the poststarburst condition, and the spatial distribution of young stars compared to the molecular gas show that the sites of current SF have changed drastically, with a $2 \mathrm{kpc}$ SF disk in the past to a $>100 \mathrm{pc}$ disk presently. Although the evidence supports the suggestion that NGC 1266 is transitioning to a poststarburst system, the presence of strong $\mathrm{H} \alpha$ emission would likely disqualify NGC 1266 from being classified as a K+A galaxy in a standard poststarburst search, since copious $\mathrm{H} \alpha$ emission is typically associated with current SF. However, Davis et al. (2012) showed that the ionized emission in NGC 1266, in particular when investigating the resolved $\log \left(\left[\mathrm{O}_{\mathrm{III}}\right] \lambda 5007 /\right.$ $\mathrm{H} \beta$ ) versus $\log ([\mathrm{S} \mathrm{II}] \lambda 6717,6731 / \mathrm{H} \alpha)$ diagnostic, lies in the LINER region of Kewley et al. (2006) and so cannot be primarily from SF.

The integral-field unit (IFU) spectra from SAURON and the GMOS IFU on the Gemini North Telescope presented in Davis et al. (2012) revealed $\mathrm{H} \alpha, \mathrm{H} \beta$, [O III], [O II], [N II], and [S II] emission within the central $2 \mathrm{kpc}$ of the galaxy. Spatial maps, line ratios, and the high velocities of the ionized gas emission were all the most consistent with a shock origin, and Davis et al. (2012) argued that the majority of $\mathrm{H} \alpha$ emission seen in NGC 1266 is not due to H II regions from SF but instead arises from shocks.
Secular processes alone, such as stellar mass-loss, will most likely not be able to significantly replenish the depleted cold gas over a short timescale and ignite any significant level of SF. This brings to light the possibility that classic searches for poststarburst galaxies are missing some exciting specimens, namely those in which an AGN is actively expelling molecular gas. If NGC 1266 is any indication, systematic searches for poststarburst galaxies should not a priori reject all galaxies with strong $\mathrm{H} \alpha$ emission. Instead, such searches should consider ratios of various ionized gas emission lines and search for indications that the emission is due to shocks rather than SF.

Given the typical timescale during which a poststarburst galaxy is detectable as such (1 Gyr; Quintero et al. 2004), 10\% of the poststarburst population could be in the process of an NGC 1266-like molecular gas expulsion (assuming a $<100 \mathrm{Myr}$ timescale for this event; Alatalo et al. 2011). A significant fraction of these poststarburst galaxies with outflows would be rejected from standard searches due to the presence of $\mathrm{H} \alpha$ emission from shocks. Because the current state of NGC 1266 represents such an important stage in a galaxy's evolution from actively star-forming to quiescent, it is essential that we modify poststarburst search routines to include NGC 1266-like objects.

\section{DISCUSSION}

\subsection{The Connection between the Poststarburst and the Molecular Gas in the Center}

Now that it has been established that NGC 1266 hosts a large amount of molecular gas, with a nonnegligible fraction 
Table 2

NGC 1266 Stellar Population Weights

\begin{tabular}{|c|c|c|c|c|c|c|}
\hline \multicolumn{7}{|c|}{ Total Age Distributions (pPXF, MIUSCAT) } \\
\hline $\begin{array}{l}\text { Kroupa } \\
\text { Universal }\end{array}$ & Age & $\begin{array}{c}\text { Light } \\
\text { Fraction }\end{array}$ & $\begin{array}{c}\text { Mass } \\
\text { Fraction }\end{array}$ & Age & $\begin{array}{c}\text { Light } \\
\text { Fraction }\end{array}$ & $\begin{array}{c}\text { Mass } \\
\text { Fraction }\end{array}$ \\
\hline Nuclear & $<2 \mathrm{Gyr}$ & 0.49 & 0.07 & $>10 \mathrm{Gyr}$ & 0.51 & 0.93 \\
\hline Integrated & $<2 \mathrm{Gyr}$ & 0.41 & 0.08 & $>10 \mathrm{Gyr}$ & 0.59 & 0.92 \\
\hline \multicolumn{7}{|c|}{ Kroupa revised } \\
\hline Nuclear & $<2$ Gyr & 0.54 & 0.09 & $>10 \mathrm{Gyr}$ & 0.46 & 0.91 \\
\hline Integrated & $<2 \mathrm{Gyr}$ & 0.40 & 0.07 & $>10 \mathrm{Gyr}$ & 0.60 & 0.93 \\
\hline \multicolumn{7}{|c|}{ Unimodal (Salpeter) } \\
\hline Nuclear & $<2$ Gyr & 0.51 & 0.08 & $>10 \mathrm{Gyr}$ & 0.49 & 0.92 \\
\hline Integrated & $<2 \mathrm{Gyr}$ & 0.40 & 0.07 & $>10 \mathrm{Gyr}$ & 0.60 & 0.93 \\
\hline \multicolumn{7}{|c|}{ Bimodal } \\
\hline Nuclear & $<2$ Gyr & 0.50 & 0.07 & $>10 \mathrm{Gyr}$ & 0.50 & 0.93 \\
\hline Integrated & $<2$ Gyr & 0.36 & 0.06 & $>10 \mathrm{Gyr}$ & 0.64 & 0.94 \\
\hline \multicolumn{7}{|c|}{ Total A- and K-star distributions (K+A only) } \\
\hline Nuclear & A (0.3 Gyr) & 0.85 & 0.12 & K (10 Gyr) & 0.15 & 0.88 \\
\hline Integrated & A (0.3 Gyr) & 0.68 & 0.12 & K (10 Gyr) & 0.32 & 0.88 \\
\hline
\end{tabular}

Notes. The total normalized stellar contributions broken up into "young" ( $<2$ Gyr) and "old" (>10 Gyr) age bins for the nuclear and integrated spectra for NGC 1266, with both mass fractions and luminosity fractions listed and fit to MIUSCAT templates assuming four different IMFs (listed above). Although both spectra have young populations, it is clear that the stellar population in the nucleus has a larger fraction of young stars as compared with the integrated spectrum. The nonnegligible fraction of young stars in NGC 1266 is independent of IMF choice. The results from a $\mathrm{K}+\mathrm{A}$ fit are also included, showing that when restricted to two templates, NGC 1266 would be classified as a poststarburst system by Quintero et al. (2004).

of that outflowing molecular gas (Alatalo et al. 2011) as well as a poststarburst stellar population, the natural next step is examining the connection between these two properties. Many authors have suggested a causal link between the AGN and the quenched SF (Hopkins et al. 2005; Nesvadba et al. 2008; Cano-Díaz et al. 2012) with the AGN and its outflow directly quenching the SF seen in galactic disks. Although ongoing AGN feedback may be able to disrupt SF in the nucleus of NGC 1266 (Alatalo et al. 2011; Davis et al. 2012; Nyland et al. 2013), evidence that the current level of AGN feedback is responsible for the decline in SF on the $2 \mathrm{kpc}$ scales of the stellar disk is currently lacking. Alatalo et al. (2011) and Davis et al. (2012) demonstrate that the timescale that the AGN has been impacting the gas is about $3 \mathrm{Myr}$, about two orders of magnitude smaller than the age of the stars in the poststarburst disk. The short timescale of current AGN activity and poststarburst timescale simply do not agree.

Observations from green valley Sloan Digital Sky Survey (SDSS) galaxies offer another possible explanation. In SDSS, Schawinski et al. (2007) observe that galaxies with AGNs in the green valley appear to have had its most recent SF episode between $100 \mathrm{Myr}$ and $1 \mathrm{Gyr}$ prior, which seems to be what we observe in NGC 1266. Assuming that the $1 \mathrm{kpc}$ poststarburst disk were just subcritical at the time of the minor merger, it is possible that the gravitational torques from the dwarf galaxy would be able to trigger a starburst while simultaneously funneling the molecular gas into the nucleus. From Cappellari et al. (2013), we know that the total stellar mass within a $1 \mathrm{kpc}$ radius is $\approx 3.9 \times 10^{9} M_{\odot}$. The young mass fraction from the integrated pPXF fit is $8 \%$ (assuming the Kroupa universal IMF).

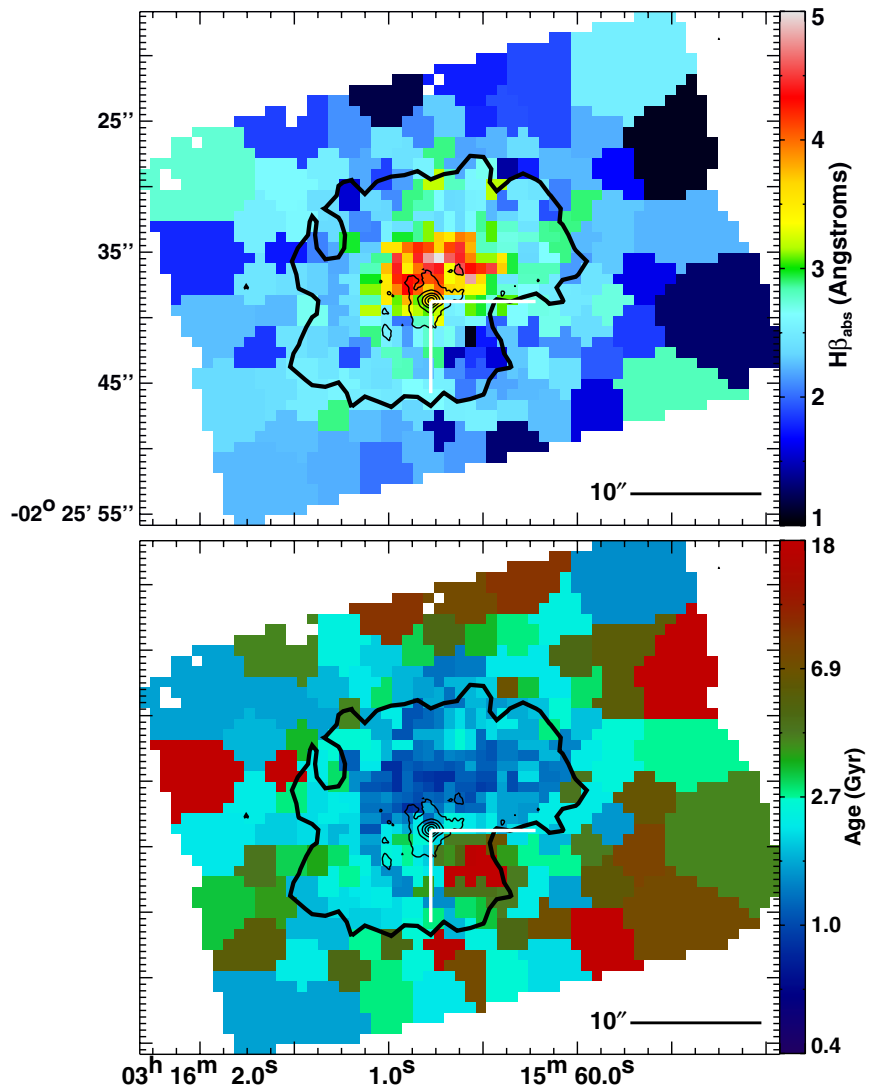

Figure 3. Top: SAURON map of the $\mathrm{H} \beta$ absorption. All absorption measurements plotted have equivalent widths $>1.4 \AA$ indicating young stars. Overlaid are both the $3 \sigma$ UV boundary (outermost contour) and the $\mathrm{CO}(1-0)$ image from Figure 4 (inner contours). Bottom: SAURON SSP model age of the stars within NGC 1266 (R. M. McDermid et al. 2014, in preparation), in logarithmic scale. It is clear from these data that the young stellar population within the galaxy is more extended than the sites of current star formation. The $U V$ image, the $\mathrm{H} \beta$ absorption map, and the SSP-derived age map show a much larger region of young stars, closer to a few kiloparsecs, than the molecular gas, likely indicating that SF has migrated inward. It is of note that the $\mathrm{H} \beta$ absorption along the axis of the outflow (approximately to the southeast of the $\mathrm{CO}$ emission) could be filled in by strong ionized gas emission (see Davis et al. 2012). White lines represent the approximate vertices of the outflow defined by the obscuration seen in the HST B-band image (Nyland et al. 2013) and are places where strong shock emission are found in Davis et al. (2012). The correspondence between the lack of $\mathrm{H} \beta$ absorption and placement of the outflow vertex argues that these decrements are likely due to extinction, rather than an intrinsic asymmetry in the young stellar populations.

This means that there is a total poststarburst stellar mass of $\approx 3.1 \times 10^{8} M_{\odot}$. The current mass of molecular gas in the system $\left(1.7 \times 10^{9} M_{\odot}\right.$; Alatalo et al. 2011) is a factor of five larger. The light fraction weighting seen in Figures 1 and 2 seems to indicate that the young stellar population is older (about $0.8 \mathrm{Gyr}$ ) at $1 \mathrm{kpc}$ than at $100 \mathrm{pc}$, indicating that it is possible that the youngest stars are closest to the nucleus. This might mean that instead of the poststarburst being driven directly by the AGN, a dynamical event 500 Myr ago was responsible both for the poststarburst population seen today, as well as for driving the molecular gas into the nucleus, which is currently being impacted by the AGN.

\subsection{Triggering Gas Inflow to the Nucleus}

The most common trigger invoked when discussing SF quenching is a major merger, but in NGC 1266 this scenario is highly unlikely. NGC 1266 does not show evidence of a major 


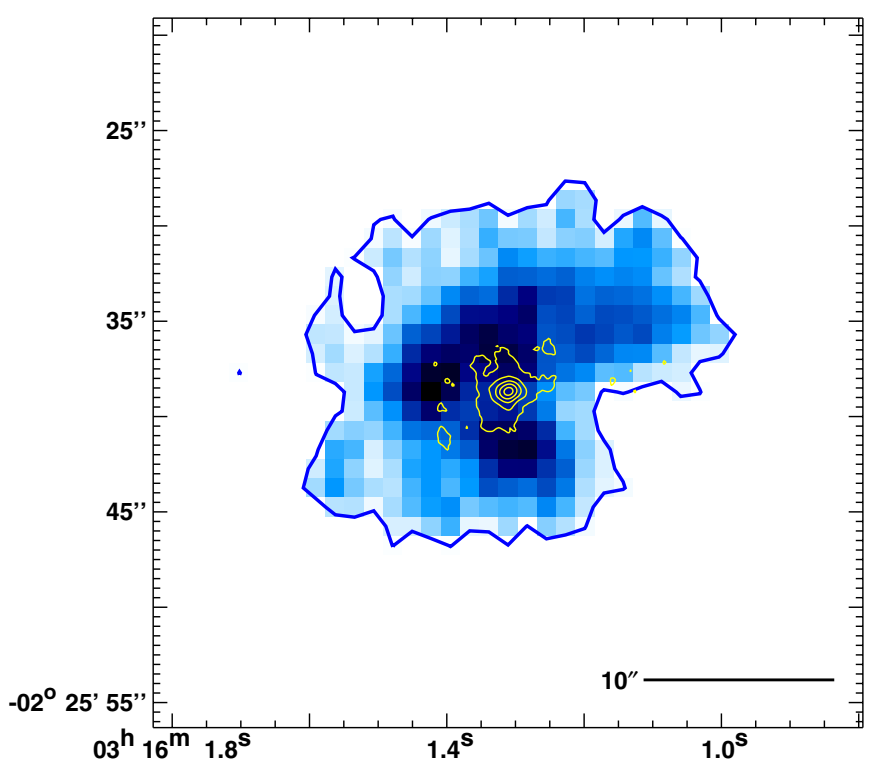

Figure 4. Swift UVOT $U V$ image (bluescale), with the $3 \sigma$ boundary appearing as a blue contour, overlaid with the $\mathrm{CO}(1-0)$ integrated intensity map (moment0; yellow contours). The $U V$ image indicates the region that contains young (but not nascent) stars, compared to the current location of the molecular gas. The total extent of the $\mathrm{CO}$ is $\approx 250 \mathrm{pc}$, with the most compact molecular gas located only in the central $100 \mathrm{pc}$ (Alatalo et al. 2011). The young stars, as traced by the $U V$, are more extended, up to $12^{\prime \prime}$, or $2 \mathrm{kpc}$.

(A color version of this figure is available in the online journal.) merger, either kinematically or morphologically. The stellar kinematics of NGC 1266 were mapped as part of the ATLAS ${ }^{3 D}$ project (Krajnović et al. 2011) and are undisturbed. Near-IR $(Y-J-H)$ images of NGC 1266 from the HST do not show any significant morphological disturbance, as one would expect from a major merger, although they do show a weak stellar spiral (Figure 5). The lack of morphological and kinematic disturbances places a constraint on the possible triggers for the poststarburst, eliminating major mergers (those with a mass ratio of more than $\approx 9: 1$; Lotz et al. 2010) within the last 1 Gyr.

On the other hand, mild gravitational encounters are capable of exciting long-lived ( $\gtrsim 1$ Gyr) spirals (Chang \& Chakrabarti 2011; Struck et al. 2011), similar to the spiral imprint seen in Figure 5. Chang \& Chakrabarti (2011) show that a perturbing companion as small as 1:100 is capable of creating the spiral pattern seen, though Struck et al. (2011) argue that the encounter should be at least a 1:10 mass ratio, which also has the added benefit that it is capable of driving the molecular gas into the center (Mihos \& Hernquist 1994; Chang 2008; Struck et al. 2011). High-sensitivity, wide-field MEGACAM $g$ and $r$ imaging of NGC 1266 are presented in Figure 6. The MEGACAM image was taken as part of the MATLAS survey. ${ }^{31}$ The acquisition and analysis of the MATLAS galaxies is identical to that described for the New Generation Virgo Survey (Ferrarese et al. 2012) and described for NGC 4216 by Paudel et al. (2013). Figure 6 seems to exhibit a weak tidal stream that is seen to the lower left of the galaxy, which could very well be the remnants

31 http://irfu.cea.fr/Projets/matlas/
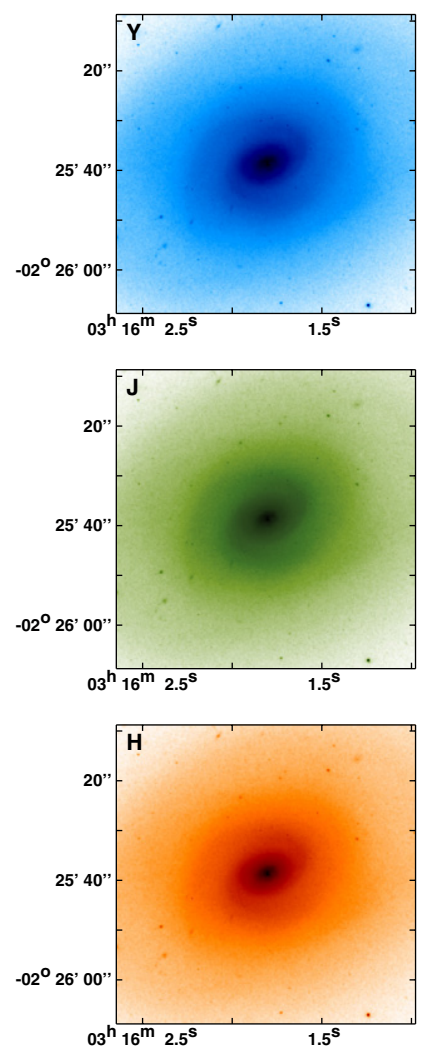
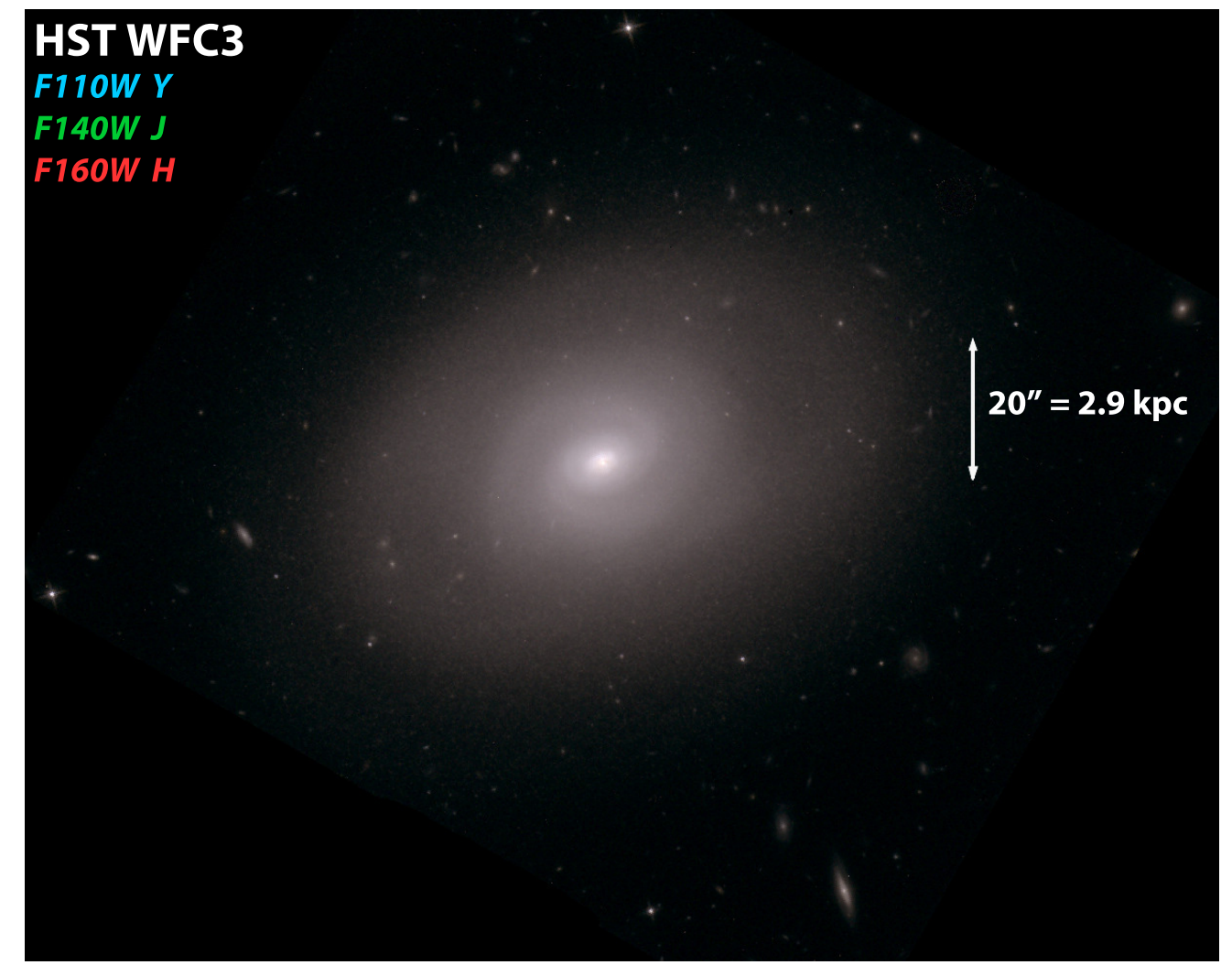

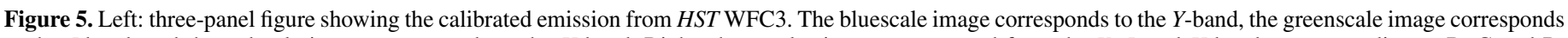

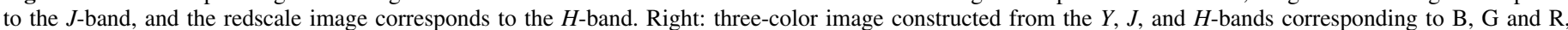

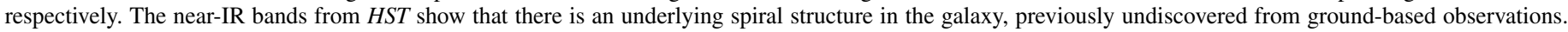

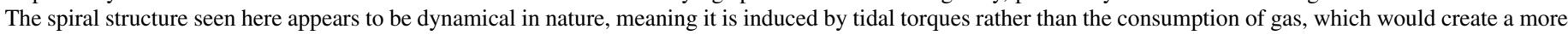
blue color to the spirals.

(A color version of this figure is available in the online journal.) 


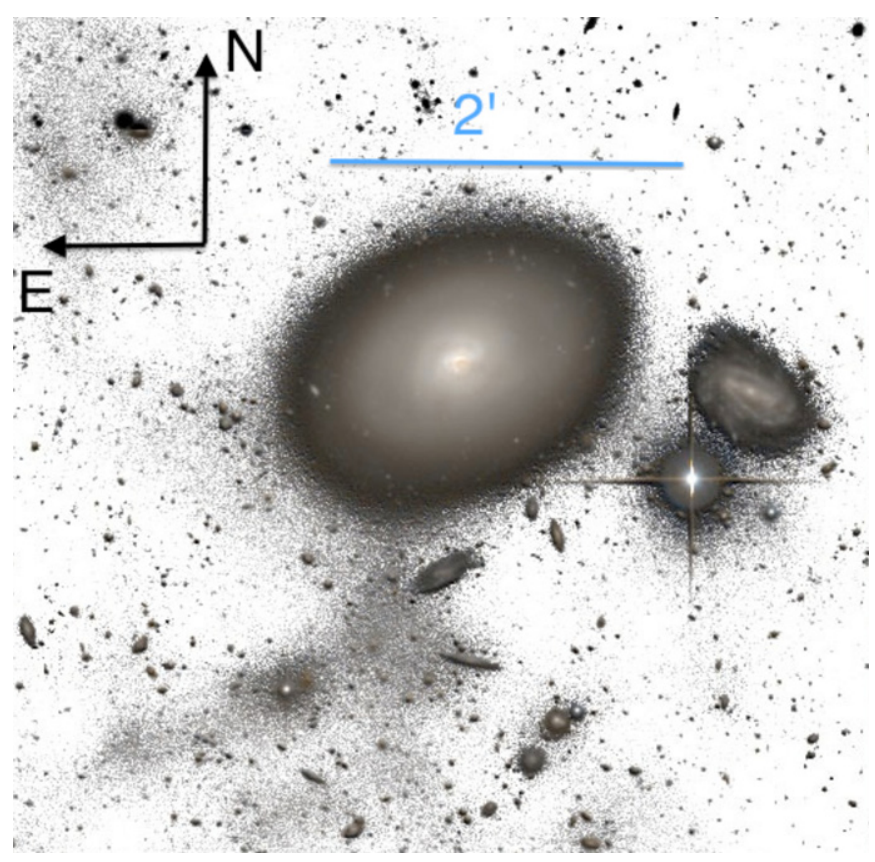

Figure 6. $g$ and $r$ wide-field imaging of NGC 1266 taken with the MEGACAM instrument on the Canada-France-Hawaii Telescope on Mauna Kea as part of the MATLAS survey (Duc et al. 2011; Paudel et al. 2013). The image shown has a limiting surface brightness of $28.5 \mathrm{mag} \operatorname{arcsec}^{-2}$. Field-of-view is $5.4 \times 5.4$ (or $47 \times 47 \mathrm{kpc}^{2}$ ). There are possibly faint tidal streams seen below and to the left of the galaxy due to material from a disrupted dwarf, but it is clear from these data that there is no sign of a major interaction in the past Gyr. The largescale feature seen to the south is likely part of a large Galactic cirrus complex. Spectroscopy of the spiral galaxy to the east shows it to be in projection in the background (J. Silverman 2012, private communication).

(A color version of this figure is available in the online journal.)

of a disrupted dwarf galaxy, as well as evidence that it is able to trace the spiral structure seen in Figure 5 to larger radii, which is visible at least $10 \mathrm{kpc}$ from the center. It is therefore possible that the spiral structure seen in Figure 5 and the faint tidal tail in Figure 6 could be imprints of a minor merger event that triggered the poststarburst and shepherded the remaining molecular gas into the center of NGC 1266 about 500 Myr ago.

If both the poststarburst and the nuclear gas are indeed connected by a common trigger, then we must explain why the molecular gas still remains in the nucleus, when one event occurred in the past 1/2 Gyr and the AGN outflow has only existed for the past 2.6 Myr (Alatalo et al. 2011). One can imagine that there might be a lag time between the ignition of the AGN and the funneling of gas into the nucleus, with many suggested mechanisms able to stall the AGN. One hypothesis is that the transport of gas from large radii forces the gas to assume some distribution in angular momentum, i.e., the stochastic model of AGN fueling (King \& Pringle 2007; Nayakshin \& King 2007). Another possibility is that cloud-cloud collisions are enhanced at small radii as initially suggested by Shlosman et al. (1990). A radiation pressure supported disk has also been suggested by Thompson et al. (2005; see the discussion in Chang 2008). Another option is bottlenecking: competition of fuel between SF and a black hole, whose mechanism for fueling at the parsec-level scales is likely due to internal instabilities (Alexander \& Hickox 2012). Despite the reconciliability of the AGN ignition time and the poststarburst event, the fact that the molecular gas has survived $500 \mathrm{Myr}$ without being completely turned into stars requires further thought.

\subsection{Sustainability of the Nuclear Molecular Gas}

Assuming the "normal" SF efficiency calculated in Leroy et al. (2008) of $5.25 \times 10^{-10} \mathrm{yr}^{-1}$, we would expect NGC 1266 to form at least $10^{9} M_{\odot}$ worth of stars within $500 \mathrm{Myr}$, thus exhausting most of the remaining $\mathrm{H}_{2}$ fuel. Given that the surface density of the nuclear gas $\left(2.7 \times 10^{4} M_{\odot} \mathrm{pc}^{-2}\right.$; Alatalo et al. 2011 ) is larger than in a normal galaxy by over two orders of magnitude, we in fact would expect that NGC 1266 would have exhausted its nuclear fuel even more efficiently if it had been sitting at this density within $100 \mathrm{pc}$ of the nucleus for the past 500 Myr. Stalling SF in the hypercompact molecular disk would therefore require an injection of energy designed to keep the disk stable against gravitational collapse to explain the gas disk's sustained existence. There seems to be evidence of a population of just such sources, with largely suppressed SF rates, namely, radio galaxies (Okuda et al. 2005; Ogle et al. 2007, 2010; Papadopoulos et al. 2008; Nesvadba et al. 2010; Guillard et al. 2012). Nesvadba et al. (2010) show that radio galaxies as a group are inefficient starformers, with efficiencies 10 to 50 times smaller than in normal galaxies. They go on to hypothesize that the turbulent energy injection from the radio jet is able to stall $\mathrm{SF}$ in the molecular gas for $\approx 10^{8} \mathrm{yr}$ (as compared to the radio jet time, of $\approx 10^{7} \mathrm{yr}$ ). This orderof-magnitude difference in timescales is explained through a cascading deposition of turbulent energy from large scales to small scales, originally suggested for the shocked region of Stephan's Quintet by Guillard et al. (2009). This would allow the molecular gas in the nucleus to remain SF inefficient for $\gtrsim 100$ Myr timescales if ignition and momentum transfer of the radio jet occurs periodically.

Recent VLBA findings of Nyland et al. (2013) show that NGC 1266 has a compact radio source, which can be attributed most likely to the AGN. The radio lobes originally described in Baan \& Klöckner (2006) are suggested as being part of a radio jet in Alatalo et al. (2011), Davis et al. (2012), and Nyland et al. (2013). This could mean that NGC 1266 is a radio galaxy whose jet just turned on (about 3 Myr ago; Alatalo et al. 2011). Schoenmakers et al. (2000) have shown that many radio jets in galaxies are cyclical phenomena, fading about $\sim 10^{7} \mathrm{yr}$ after the initial jet creation. This would mean that the radio jet is able to impact the molecular gas $\sim 10$ times longer than it is visible. It is therefore possible that the radio jet in NGC 1266 could be a cyclical phenomenon, leaving an impact on the SF in the molecular disk without the outward signs of radio synchrotron lobes (which have already faded). In fact, a $365 \mathrm{MHz}$ radio survey conducted by Douglas et al. (1996) indicates that NGC 1266 appears to have be double lobed structure, $25^{\prime \prime}$ ( or $\approx 4 \mathrm{kpc}$ ) in size, at least twice as large as the $1.4 \mathrm{GHz}$ detected radio jets (Alatalo et al. 2011; Nyland et al. 2013). This conclusion was reached by fitting the interferometric data to a model; therefore it is important to confirm the existence of the low frequency double-lobed jet through radio imaging.

If the radio jet feedback seen in NGC 1266 is indeed cyclical, then gas is currently being heated and deposited into the galactic halo, which is commonly seen in galaxy clusters and groups (Gitti et al. 2011, and references therein). In most cases where $\mathrm{X}$-ray bubbles are seen, the gas is being deposited into the media of either a group or a cluster. NGC 1266 appears to be isolated (Cappellari et al. 2011b) and therefore does not have access to the combined gravitational potential of many nearby neighbors. Boroson et al. (2011) do confirm that hot gas haloes are present around isolated (and less massive) galaxies, but at a much lower luminosity. This is likely because much of the gas is able to 
escape the gravitational potential of the galaxy and therefore would be unavailable for future recycling. One conclusive way to confirm whether or not the SF quenching episode is due to this cyclical AGN feedback mechanism is with deep X-ray and low frequency radio observations to search for fossil shells from previous episodes, noting the $365 \mathrm{MHz}$ interferometric data provide compelling evidence that this may be the case for NGC 1266.

It is also possible that these two events, the $500 \mathrm{Myr}$ old post-starburst and the current expulsion of the molecular gas, are not related at all. If this is the case, there are many scenarios that are able to explain the poststarburst event, including the previously discussed gravitational encounter or possibly an AGN event similar to what is hypothesized in Cano-Díaz et al. (2012) in which the AGN in the system is directly creating the poststarburst. The nuclear molecular gas, on the other hand, remains very difficult to explain, as a minor merger capable of depositing all $1.7 \times 10^{9} M_{\odot}$ of gas into the center would have to be at least a 5:1 ratio and have deposited its gas less than a couple dynamical times ago, which should mean leaving sufficient evidence behind. Instead, there is little evidence of this, both from the lack of H I emission (Alatalo et al. 2011; Nyland et al. 2013) as well as the lack of prominent tidal features (Figure 6).

Given the observational evidence, it seems that the current favored explanation for the current state of molecular gas is that a gravitational encounter $500 \mathrm{Myr}$ ago was able to drive the molecular gas into the nucleus, prompting first a starburst, then quenching SF within the $1 \mathrm{kpc}$ disk. The spiral structure seen in Figures 5 and 6 supports this picture. The torques also caused the molecular gas to funnel into the nucleus, where the AGN was able to inhibit its ability to form stars through the injection of turbulence from the radio jets.

\section{SUMMARY AND CONCLUSIONS}

We report new observations of NGC 1266, a local example of an AGN-driven molecular outflow and a candidate for AGN-driven SF quenching.

An investigation of the spatial distribution of young stars using the Swift UVOT $U V$-band as well as SAURON stellar absorption and age maps, compared with the molecular gas distribution, shows that the sites of current SF are far more compact than the regions containing young stars. This points to an outward-in cessation of SF within the galaxy and suggests that NGC 1266 might be transitioning into a poststarburst object.

We also performed a stellar population analysis of NGC 1266 utilizing the spectra from Moustakas \& Kennicutt (2006). The absorption features in the nucleus of NGC 1266 indicate the presence of a nonnegligible fraction of $<1$ Gyr aged stars. The model-derived A/K fraction of NGC 1266 of 2.1 would lead it to be classified as poststarburst in SDSS, but previous studies have likely failed to recognize the poststarburst nature of NGC 1266 due to the presence of strong ionized gas emission. However, as Davis et al. (2012) demonstrated, the ionized gas in NGC 1266 is most likely the result of shocks associated with the outflow rather than SF. NGC 1266-like poststarbursts may be rejected by standard poststarburst searches due to the presence of the ionized gas emission, and it is therefore imperative to expand the search for poststarburst candidates to include galaxies with shock-like line ratios.

The poststarburst stellar population within NGC 1266 sets a timescale for the quenching event of $\approx 500 \mathrm{Myr}$ ago, which seems to indicate that current AGN activity cannot be directly responsible for the cessation of SF in the $2 \mathrm{kpc}$ disk. Instead, it is possible that a gravitational encounter, which excited spirals within the galaxy, was able to drive the molecular gas into the center, thus quenching any possible SF outside of $\approx 100 \mathrm{pc}$ of the nucleus. Recent studies of radio galaxies seem to indicate that the AGN then might be able to prevent the nuclear molecular gas from forming stars by injecting turbulent kinetic energy via a periodical ignition of the radio jet.

K.A. would like to thank Carl Heiles, Phil Appleton, and Joan Wrobel for many useful discussions and input, as well as John Moustakas for access to the most up-to-date version of the long-slit spectra. K.A. would also like to thank the anonymous referee for prompt replies and insightful suggestions that have vastly improved this article. The research of K.A. is supported by the NSF grant AST-0838258 and by NASA grant HST-GO-12526. K.A. was also partially supported by funding through Herschel, a Europian Space Agency Cornerstone Mission with significant participation by NASA, through an award issued by JPL/Caltech. K.N. is supported by NSF grant 1109803. S.L.C. was supported by ALMA-CONICYT program 31110020. P.S. is an NWO/Veni fellow. R.M.McD. is supported by the Gemini Observatory, which is operated by the Association of Universities for Research in Astronomy, Inc., on behalf of the international Gemini partnership of Argentina, Australia, Brazil, Canada, Chile, the United Kingdom, and the United States of America. R.L.D. and M.B. are supported by the rolling grants Astrophysics at OxfordPP/E001114/1 and ST/ H002456/1 from the UK Research Councils. R.L.D. acknowledges travel and computer grants from Christ Church, Oxford and support from the Royal Society in the form of a Wolfson Merit Award 502011.K502/jd. T.N. acknowledges support from the DFG Cluster of Excellence: Origin and Structure of the Universe. Observations were made with the NASA/ESA Hubble Space Telescope, obtained from the Space Telescope Science Institute, which is operated by the Association of Universities for Research in Astronomy, Inc., under NASA contract NAS 5-26555. These observations are associated with program 12526. This work has made use of the tools and services available through the HEASARC online service, which is operated by the Goddard Space Flight Center for NASA. We acknowledge the use of public data from the Swift data archive.

\section{REFERENCES}

Aalto, S., Garcia-Burillo, S., Muller, S., et al. 2012a, A\&A, 537, 44 Aalto, S., Muller, S., Sakamoto, K., et al. 2012b, A\&A, 546, 68 Alatalo, K., Blitz, L., Young, L. M., et al. 2011, ApJ, 735, 88 Alexander, D. M., \& Hickox, R. C. 2012, NewAR, 56, 93 Baan, W., \& Klöckner, H. R. 2006, A\&A, 449, 559

Bacon, R., Copin, Y., Monnet, G., et al. 2001, MNRAS, 326, 23 Baldry, I. K., Glazebrook, K., Brinkmann, J., et al. 2004, ApJ, 600, 681 Bekki, K. 1998, ApJL, 502, L133

Bekki, K., Couch, W. J., \& Shioya, Y. 2002, ApJ, 577, 651

Bell, E. F., Wolf, C., Meisenheimer, K., et al. 2004, ApJ, 608, 752 Boroson, B., Kim, D.-W., \& Fabbiano, G. 2011, ApJ, 729, 12

Boselli, A., \& Gavazzi, G. 2006, PASP, 118, 517

Breeveld, A. A., Curran, P. A., Hoversten, E. A., et al. 2010, MNRAS, 406, 1687

Cales, S. L., Brotherton, M. S., Shang, Z., et al. 2011, ApJ, 741, 106

Cano-Díaz, M., Maiolino, R., Marconi, A., et al. 2012, A\&A, 537, 8

Cappellari, M., \& Copin, Y. 2003, MNRAS, 342, 345

Cappellari, M., \& Emsellem, E. 2004, PASP, 116, 138

Cappellari, M., Emsellem, E., Krajnović, D., et al. 2011a, MNRAS, 413, 813

Cappellari, M., Emsellem, E., Krajnović, D., et al. 2011b, MNRAS, 416, 1680

Cappellari, M., Scott, N., Alatalo, K., et al. 2013, MNRAS, 432, 1709

Chang, P. 2008, ApJ, 684, 236 
Chang, P., \& Chakrabarti, S. 2011, MNRAS, 416, 618

Ciotti, L., \& Ostriker, J. P. 2007, ApJ, 665, 1038

Ciotti, L., Ostriker, J. P., \& Proga, D. 2010, ApJ, 717, 708

Croton, D. J., Springel, V., White, S. D. M., et al. 2006, MNRAS, 365, 11

da Cunha, E., Charlot, S., \& Elbaz, D. 2008, MNRAS, 388, 1595

Das, M., Vogel, S. N., Verdoes Kleijn, G. A., O’Dea, C. P., \& Baum, S. A. 2005, ApJ, 629, 757

Davis, T. A., Krajnović, D., McDermid, R. M., et al. 2012, MNRAS, 426, 1574

Debuhr, J., Quataert, E., \& Ma, C.-P. 2012, MNRAS, 420, 2221

Douglas, J. N., Bash, F. N., Bozyan, F. A., Torrence, G. W., \& Wolfe, C. 1996, AJ, 111,1945

Dressler, A., \& Gunn, J. E. 1983, ApJ, 270, 7

Duc, P. A., Cuillandre, J.-C., Serra, P., et al. 2011, MNRAS, 417, 863

Evrard, A. E. 1991, MNRAS, 248, 8

Faber, S. M., Willmer, C. N. A., Wolf, C., et al. 2007, ApJ, 665, 265

Falcón-Barroso, J., Sánchez-Blázquez, P., Vazdekis, A., et al. 2011, A\&A, 532, 95

Falkenberg, M. A., Kotulla, R., \& Fritze, U. 2009, MNRAS, 397, 1940

Ferrarese, L., Côté, P., Cuillandre, J.-C., et al. 2012, ApJS, 200, 4

Feruglio, C., Maiolino, R., Piconcelli, E., et al. 2010, A\&A, 518, L155

Fischer, J., Sturm, E., González-Alfonso, E., et al. 2010, A\&A, 518, L41

Fujita, Y. 1998, ApJ, 509, 587

Gitti, M., Brighenti, F., \& McNamara, B. R. 2011, AdAst, 2012, 950641

Goto, T. 2005, MNRAS, 357, 937

Guillard, P., Boulanger, F., Pineau des Forêts, G., \& Appleton, P. N. 2009, A\&A, 502,515

Guillard, P., Ogle, P. M., Emonts, B. H. C., et al. 2012, ApJ, 747, 95

Hopkins, P. F., Hernquist, L., Cox, T. J., et al. 2005, ApJ, 630, 705

Hota, A., Rey, S.-C., Kang, Y., et al. 2012, MNRAS, 422, 38

Icke, V. 1985, A\&A, 144, 115

Kennicutt, R. C. 1998, ARA\&A, 36, 189

Kennicutt, R. C., Armus, L., Bendo, G., et al. 2003, PASP, 115, 928

Kewley, L. J., Groves, B., Kauffmann, G., \& Heckman, T. 2006, MNRAS, 372,961

King, A. R., \& Pringle, J. E. 2007, MNRAS, 377, L2

Krajnović, D., Emsellem, E., Cappellari, M., et al. 2011, MNRAS, 414, 2923

Kroupa, P. 2001, MNRAS, 322, 231
Leroy, A. K., Walter, F., Brinks, E., et al. 2008, AJ, 136, 2782

Lotz, J. M., Johnsson, P., Cox, T. J., \& Primack, J. R. 2010, MNRAS, 404, 575

Martig, M., Bournaud, F., Teyssier, R., \& Dekel, A. 2009, ApJ, 707, 250

Matsushita, S., Muller, S., \& Lim, J. 2007, A\&A, 468, 49

Mihos, J. C. 1995, ApJL, 438, L75

Mihos, J. C., \& Hernquist, L. 1994, ApJL, 425, L13

Moore, B., Katz, N., Lake, G., Dressler, A., \& Oemler, A. 1996, Natur, 379,613

Moustakas, J., \& Kennicutt, R. C. 2006, ApJS, 164, 81

Nayakshin, S., \& King, A. 2007, arXiv:0705.1686

Nesvadba, N. P. H., Boulanger, F., Salom, P., et al. 2010, A\&A, 521, A65

Nesvadba, N. P. H., Lehnert, M. D., De Breuck, C., Gilbert, A. M., \& van Breugel, W. 2008, A\&A, 491, 407

Nyland, K. E., Alatalo, K., Wrobel, J. M., et al. 2013, ApJ, 779, 173

Ogle, P., Antonucci, R., Appleton, P. N., \& Whysong, D. 2007, ApJ, 668, 699

Ogle, P., Boulanger, F., Guillard, P., et al. 2010, ApJ, 724, 1193

Okuda, T., Kohno, K., Iguchi, S., \& Nakanishi, K. 2005, ApJ, 620, 673

Papadopoulos, P. P., Kovacs, A., Evans, A. S., \& Barthel, P. 2008, A\&A, 491, 483

Paudel, S., Duc, P.-A., Côté, P., et al. 2013, ApJ, 767, 133

Quintero, A. D., Hogg, D. W., Blanton, M. R., et al. 2004, ApJ, 602, 190

Roming, P. W. A., Kennedy, T. E., Mason, K. O., et al. 2005, SSRv, 120, 95

Salpeter, E. E. 1955, ApJ, 121, 161

Sarzi, M., Falcón-Barroso, J., Davies, R. L., et al. 2006, MNRAS, 366, 1151

Schawinski, K., Thomas, D., Sarzi, M., et al. 2007, MNRAS, 382, 1415

Schoenmakers, A. P., de Bruyn, A. G., Röttering, H. J. A., van der Laan, H., \& Kaiser, C. R. 2000, MNRAS, 315, 371

Shlosman, I., Begelman, M. C., \& Frank, J. 1990, Natur, 345, 679

Springel, V., Di Matteo, T., \& Hernquist, L. 2005, MNRAS, 361, 776

Strateva, I., Ivezić, Ž., Knapp, G. R., et al. 2001, AJ, 122, 1861

Struck, C., Dobbs, C. L., \& Hwang, J. S. 2011, MNRAS, 414, 2498

Thompson, T. A., Quataert, E., \& Murray, N. 2005, ApJ, 630, 167

Vazdekis, A., Ricciardelli, E., Cenarro, A. J., et al. 2012, MNRAS, 424, 157

Vazdekis, A., Sánchez-Blázquez, P., Falcón-Barroso, J., et al. 2010, MNRAS, 404,1639

Verdes-Montenegro, L., Yun, M. S., Williams, B. A., et al. 2001, A\&A, 377,812

Zabludoff, A. I., Zaritsky, D., Lin, H., et al. 1996, ApJ, 466, 104 